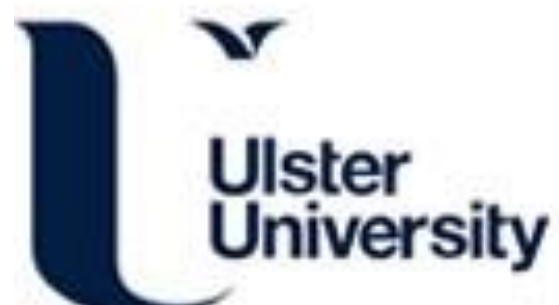

\section{Assessment of an osteoblast-like cell line as a model for human primary osteoblasts using Raman spectroscopy}

McManus, LL., Bonnier, F., Burke, GA., Meenan, BJ., Boyd, A., \& Byrne, H. (2012). Assessment of an osteoblast-like cell line as a model for human primary osteoblasts using Raman spectroscopy. ANALYST, 137(7), 1559-1569. https://doi.org/10.1039/C2AN16209A

Link to publication record in Ulster University Research Portal

Published in:

ANALYST

Publication Status:

Published (in print/issue): 21/02/2012

DOI:

10.1039/C2AN16209A

Document Version

Author Accepted version

\section{General rights}

Copyright for the publications made accessible via Ulster University's Research Portal is retained by the author(s) and / or other copyright owners and it is a condition of accessing these publications that users recognise and abide by the legal requirements associated with these rights.

\section{Take down policy}

The Research Portal is Ulster University's institutional repository that provides access to Ulster's research outputs. Every effort has been made to ensure that content in the Research Portal does not infringe any person's rights, or applicable UK laws. If you discover content in the Research Portal that you believe breaches copyright or violates any law, please contact pure-support@ulster.ac.uk. 


\title{
Assessment of an osteoblast-like cell line as a model for human primary osteoblasts using Raman spectroscopy $\dagger$
}

\author{
Lindsay L. McManus, ${ }^{* a}$ Franck Bonnier, ${ }^{b}$ George A. Burke, ${ }^{a}$ Brian J. Meenan, ${ }^{a}$ Adrian R. Boyd ${ }^{a}$ \\ and Hugh. J. Byrne ${ }^{b}$
}

Received 5th December 2011, Accepted 1st February 2012

DOI: 10.1039/c2an16209a

Raman spectroscopy is employed to determine the suitability of the U20S osteoblast-like cell line for use as a model for human primary osteoblasts, with emphasis on the ability of these cell types to replicate their tissue of origin. It was found that both cell types demonstrated early stage mineral deposition that followed significantly different growth patterns. Analysis of the growth pattern and spectral data from primary cells revealed increasing bone quality ratios and a high crystallinity, consistent with previous reports. Conversely the investigation of the U20S osteoblast-like cell line provided evidence of dense multilayered mineralised regions that corresponded more closely to native bone in terms of its crystallinity and bone quality ratios. This finding contradicts previous reports on U20S osteoblast-like cells which have consistently described them as non-osteoinductive when cultured in various conditions on a number of substrates. This work demonstrates the successful application of Raman spectroscopy combined with biological and multivariate analysis for the investigation of osteoblast-like U20S cells and human primary osteoblasts, specifically with focus on the osteoinductive ability of the osteoblast-like cell line and the comparative differences in relation to the primary osteoblasts.

\section{Introduction}

When investigating diseases such as cancer, one point of focus is on the identification of drugs that have a positive influence on metabolic uptake, cell differentiation and the induction of cell death. Stem cells are now commonly being used in regenerative medicine and tissue engineering applications with the primary aim of replacing or repairing tissue that has been diseased or damaged. ${ }^{1}$ The fulfilment of this aim relies heavily on understanding cell behaviour and cell interaction with other tissues and materials both in vitro and in vivo. From this perspective, primary cells and lineage-specific cells are the gold standard when studying live cell interactions in vitro. With particular relevance to bone tissue engineering, de novo bone can be produced by culturing bone cells onto different biomaterials. Osteoblasts initiate bone formation by producing osteoid prior to the mineralisation process. Osteoid is an unmineralised intracellular matrix composed of collagen fibrils and fibres combined with

${ }^{a}$ Engineering Research Institute, Nanotechnology and Integrated BioEngineering Centre (NIBEC), University of Ulster, BT37 OQB, Northern Ireland. E-mail: linzimcmanus@gmail.com; Fax: +44 (0) 2890 366863; Tel: + 44 (0) 2890368928

${ }^{b}$ Focas Research Institute, Dublin Institute of Technology (DIT), Kevin Street, Dublin 8, Ireland

$\dagger$ Electronic supplementary information (ESI) available. See DOI: $10.1039 / \mathrm{c} 2 \mathrm{an} 16209 \mathrm{a}$ a proteinaceous component that mineralises to become bone. As a result, osteoblasts are essential for the process of bone remodelling and play a crucial role in healing various bone diseases. ${ }^{2-6}$ Primary cells are traditionally used when studying live cell interactions and, in this respect, primary osteoblast cells isolated directly from the patient are the ideal model when investigating the formation of new bone. However, their use is restricted due to the difficulty faced when isolating bone fragments that are capable of producing cells that can be harvested in sufficient numbers. Primary osteoblasts are also challenging to expand in vitro and with increasing passage number they can lose their phenotype in a process known as de-differentiation, leading to a finite life-span..$^{7-9}$ More recently, Rubio et al. have reported the spontaneous transformation of adult stem cells with increasing passage number, therefore indicating the safety concerns when expanding cells in large numbers. ${ }^{10}$

To overcome these limitations, cell lines are often used as an alternative model for primary cells and, to date, many cell line models have been chosen because they have a longer life-span than primary cells. They can also proliferate indefinitely, whilst maintaining phenotypic stability, therefore overcoming the limitations of using primary cells. These cell lines are routinely used to provide an understanding that reflects the interaction of osteoblasts with various materials, ${ }^{11-14}$ and are typically obtained by the immortalization of primary cells through transduction or retroviral transfection or, in the current study, from cancerous 
osteosarcoma tissue. However, the use of osteoblast-like cell lines presents a major potential drawback, which is that the phenotype expressed may not be a true representation of the phenotype of primary osteoblasts. ${ }^{15,16}$

Human osteoblast-like cell lines have been used in bone tissue engineering as models for investigating primary osteoblast function and they are used regularly to study growth, integral expression, cell function and the effects of cytokines and growth factors. Bilbe et al. reported that three osteoblast-like cell lines were highly comparable with primary osteoblasts. This finding was based on the similar expressions of a total of 58 cytokines, growth factors and bone related proteins from the cell lines and primary cells. ${ }^{15}$ Conversely, Clover et al. found that, depending on the osteoblastic function being investigated, osteoblast-like cell lines could be used as appropriate models of primary cells. Osteoblast-like cell lines were concluded to be ideal models for investigating integrin expression, cell adhesion and the production of osteocalcin. However, in terms of alkaline phosphatase expression and cell proliferation, osteoblast-like cells were not a good representation of primary cells. ${ }^{16}$

Various studies have reported the use of osteoblast-like cells derived from osteosarcoma and have established the relevance of their use to study in vitro interactions with biomaterials, ${ }^{17-19}$ bone cell mineralisation, ${ }^{20,21}$ and regarding their suitability for a stable representation of their primary counterparts. ${ }^{7}$ Using Raman spectroscopy, similarities in phenotype and biochemical composition have been found, as well as many differences between primary cells and the various sarcoma derived cell lines, including the decrease in the concentration of nucleic acids in sarcoma derived cells and the increase in protein concentrations in primary cells. ${ }^{7}$

Various biological, univariate and multivariate techniques have been employed in parallel with Raman spectroscopy to provide a comprehensive comparison of the osteoinductive potential of human primary osteoblasts (HOBs) and osteoblastlike U20S cells (U20Ss). This paper determines the suitability of the U20S osteoblast-like cell line as an alternative to primary osteoblast cells. It presents spectral data from primary cells which indicate increasing bone quality ratios and a high crystallinity with culture time. Similarly, there is evidence of a bone material produced by U20Ss that is comparable to native bone with respect to its crystallinity and bone quality markers. This in itself is a very interesting finding, Due to the apparent nonosteoinductive ability of this cell type..$^{22-24}$

\section{Materials and methods}

\section{Primary cell culture}

Primary HOBs derived from cancellous bone pieces (Promocell, UK) were used in this study. Isolation is performed by the manufacturer. Briefly, cancellous bone was cut into small pieces that are approximately $2-4 \mathrm{~mm}$ in width with appropriate bone scissors. The pieces were washed and cultivated on a plastic flask with the manufacturer osteoblast growth medium. After 1-3 weeks, the osteoblasts grow out of the bone pieces and proliferate. Cells were maintained in osteoblast basal media (product number C-27001B) and supplemented with osteoblast supplement mix (product number C39616) which includes ascorbic acid and foetal bovine serum.

Osteogenic differentiation was induced by culturing HOBs in osteoblast mineralisation medium (product number C-27020), supplemented with osteogenic differentiation medium supplement mix (product number C-39616) which contains ascorbic acid, beta-glycerophosphate, dexamethasone and foetal bovine serum. All cells were maintained below $70 \%$ confluency and were passaged every three to four days using $0.05 \%$ trypsin-EDTA. All chemicals were purchased from Promocell, UK. HOBs were seeded at a density of approximately 30,000 cells per $\mathrm{cm}^{2}$ onto calcium fluoride $\left(\mathrm{CaF}_{2}\right)$ substrates (Crystran, UK). Cells were cultured in normal humidified conditions at $37^{\circ} \mathrm{C}, 5 \% \mathrm{CO}_{2}$ for 7 , 14, 21 and 28 days, respectively.

\section{Osteoblast-like cell culture}

U20S osteoblast-like cells cultivated from the bone tissue of a 15 year old female suffering from a moderately differentiated osteosarcoma of the tibia were used in this study (Health Protection Agency, UK). Cells were maintained in McCoy's 5A medium and supplemented with L-Glutamine and 10\% foetal bovine serum (Invitrogen, UK).

Mineralisation was induced by culturing U20Ss in osteoblast media supplemented with $10 \mathrm{nM}$ dexamethasone, $10 \mathrm{mM}$ betaglycerophosphate and $0.05 \mathrm{mM}$ ascorbic acid-2-phosphate (Invitrogen, UK). All cells were maintained at below 70\% confluency and were passaged every three to four days using $0.25 \%$ trypsin-EDTA (Sigma, UK). U20Ss were seeded at a density of 10,000 cells per $\mathrm{cm}^{2}$ onto $\mathrm{CaF}_{2}$ substrates (Crystran, UK). Cells were cultured in normal humidified conditions at $37^{\circ} \mathrm{C}, 5 \% \mathrm{CO}_{2}$ for 7, 14, 21 and 28 days, respectively.

For Raman analysis, all samples were taken at seven day intervals and fixed in $10 \%$ formalin solution for $10 \mathrm{mins}$, cells were then stored in $0.9 \%$ sodium chloride $(\mathrm{NaCl})$ for up to 3 weeks.

\section{Alizarin red staining}

To confirm the presence of mineralisation, alizarin red staining was performed at days 7, 14, 21 and 28. Cells were washed three times in PBS, fixed and permeabilised in $4 \%$ paraformaldehyde (PFA), with $0.1 \%$ Triton $\mathrm{X}-100$ for $20 \mathrm{~min}$ at room temperature then washed twice in de-ionised water to remove residual PFA. Cells were stained with $2 \%$ alizarin red for $30 \mathrm{~min}$ at room temperature with gentle shaking. For the colorimetric assay 3.6\% $(0.1 \mathrm{M})$ cetylpyridinium chloride was added to each sample for $45 \mathrm{~min}$ and the optical density was measured at $405 \mathrm{~nm}$.

\section{Raman spectroscopy}

Raman spectra of non-osteogenic and osteogenic cultured HOBs and U20Ss were obtained at seven day intervals up to 28 days using A Horiba Jobin-Yvon LabRAM HR800 UV spectrometer with a single mode diode laser operating at $660 \mathrm{~nm}$. For all spectral measurements, a x100 water immersion objective (LUMPlanF1, Olympus, N.A. $1.00 \mathrm{~W}$ ) was employed, providing a spatial resolution of $\sim 1 \mu \mathrm{m}$ at the sample. The confocal hole was set at $100 \mu \mathrm{m}$ for all measurements (the specified setting for confocal operation). The system was spectrally calibrated to the $520.7 \mathrm{~cm}^{-1}$ spectral line of Silicon. The LabRAM system is a confocal spectrometer that 
contains two interchangeable gratings (300 and 900 lines $/ \mathrm{mm}$ respectively). In the following experiments the 300 lines $/ \mathrm{mm}$ grating was employed, which has a spectral dispersion of $\sim 1.5 \mathrm{~cm}^{-1}$ per pixel. The detector used was a 16-bit dynamic range Peltier cooled CCD detector. Images of the sample were acquired using a video camera within the system.

\section{Immunocytochemistry}

After 28 days, cells were washed in large volumes of ice-cold PBS $(3 \times 5 \mathrm{~min})$, fixed and permeabilised in $4 \%$ PFA containing $0.1 \%$ Triton X-100 for $20 \mathrm{~min}$ at $4{ }^{\circ} \mathrm{C}$ and stained with bone specific proteins, including, osteocalcin, osteopontin, alkaline phosphatase and SPARC (osteonectin) (Santa Cruz Biotechnology Inc, USA). Following washing, samples were treated with a blocking solution (PBS containing 1.0\% BSA, 5\% normal goat serum (NGS)) for $30 \mathrm{~min}$ at room temperature. After blocking, the samples were washed in PBS and then incubated with the appropriate primary antibodies for $1 \mathrm{~h}$ before exposure to the opposite secondary antibodies. Following secondary antibody incubation, cells were counterstained with DAPI nucleic acid stain (Molecular Probes, Inc. Invitrogen). After washing twice in PBS, substrates were mounted in Vector shield mounting media (Vector Laboratories, UK). The substrates were sealed using a clear nail varnish and examined using a Carl Zeiss LSM5 Pascal (Carl Zeiss MicroImaging, Germany) Confocal Laser Scanning Microscope (CLSM). The diluent for all antibodies was PBS - 1\% BSA.

\section{Data pre-processing}

It is accepted that spectral artifacts can have a considerable effect on the interpretation of data. Therefore it is necessary to distinguish between biochemical information and undesired effects. With Raman spectroscopy, is it known that before data interpretation can be performed, pre-processing of data should be carried out. To this end, Matlab (Mathworks, USA) was used to routinely pre-process the raw data. In the first instance, band alignment was performed to correct for instrumental spectral shifts. As such, all spectra were normalised using the area of the $1449 \mathrm{~cm}^{-1}$ band. $\mathrm{A} \mathrm{CaF}_{2}$ background spectrum was acquired for each individual substrate used, and subsequently subtracted before averaging. A simple baseline subtraction was performed and smoothing by a 5th order, 11-point Savitzky-Golay filter.

\section{Principal component analysis}

Principal component analysis is a multivariate analysis technique that is widely used to simplify a complex data set of multiple dimensions. It allows the transformation of different sets of variables into a smaller set of new variables or principal components that capture the maximum variance in the data. The order of the principal components represents their importance to the original dataset, so the greatest variance is illustrated by the first principal component (PC1); the second greatest variance is the second principal component (PC2) and so forth. In principle, each principal component is a linear combination of the original variables for the simplified variables, therefore, var $(\mathrm{PC} 1) \geq \operatorname{var}$ $(\mathrm{PC} 2) \geq(\mathrm{PCp})$, where var $(\mathrm{PCi})$ represents the variance of PCi in the dataset. A scatter plot is generated from the data which shows groups of points representing variations within the dataset. ${ }^{25}$ PCA was performed using Matlab (Mathworks, USA) following the different data analysis steps described in the previous section. The other advantage of this method is the observation of loadings which represent the variance for each variable (wavenumber) for a given PC. Analysing the loadings of a PC can give information about the source of the variability inside a dataset, derived from variations in the molecular components contributing to the spectra.

This statistical method of analysis was preferred because it is able to resolve spectral components that are present in different proportions. This is extremely important when bone is the material being analysed, as it is heterogeneous in nature, which results in considerable variations in its spectra.

\section{Results and discussion}

\section{Cell morphology}

HOBs are widely dispersed cells with a prominent nucleus and they exhibit a typical elongated fibroblast-like morphology with a spindle-like appearance (Supplementary Figure $1 \mathrm{~A}-\mathrm{B} \dagger$ ). They have a relatively large body and were measured to be approximately $130-160 \mu \mathrm{m}$ in length and $10-20 \mu \mathrm{m}$ in diameter with an aspect ratio of $8.3 \pm 3.6$. The morphology of U20Ss differs and as such the U20Ss used during this study exhibit typical epithelial morphological characteristics. After $48 \mathrm{~h}$ in culture, there was little to no overlap observed and the cells were generally hexagonal in shape and of a 'cobblestone' appearance that are interconnected by cell processes (Supplementary Figure 1C-D $†$ ). U20S cells have a small cell body with a large cell nucleus and few cytoplasmic processes and were measured to be approximately $30-55 \mu \mathrm{m}$ in length and $25-50 \mu \mathrm{m}$ in diameter with an aspect ratio of $1.2 \pm 1.1$.

Both non-osteogenic and osteogenic HOBs cell cultures underwent differing growth patterns, as expected. As can be seen in Fig. 1, for cells grown in non-osteogenic media (Fig. 1A), there was no evidence of mineral formation. Non-osteogenic HOBs maintained a fibroblast-like morphology with a spindle-like shaped cell body throughout the 28 day culture period. Fig. 1A shows the control of HOBs samples cultured in non-osteogenic media, HOBs cultured in osteogenic media for 7 days (Fig. 1B) highlights a change in growth pattern. When cultured for 7 days in media with osteogenic supplements, whilst no calcium deposition or mineral formation is observed, there is a dramatic change in cell morphology. HOBs have not maintained the elongated fibroblast appearance that is observed in Fig. 1A, instead they have become more cuboidal in shape and have formed a dense monolayer during the 7 days in culture. At day 14 , HOBs appear more rounded in shape and the monolayer has become thicker. Cell/protein aggregation within the monolayer is also observed at this culture time point, as shown in Fig. 1C. Dramatic changes in cell morphology are observed between days 14-21. Fig. 1C and Fig. 1D show that between these culture times, the cell monolayer became patchy and in the outer regions of the cell monolayer, there are discrete areas of calcium deposition and bone nodule formation. By day 28, as shown in Fig. 1E the outer region of the cell monolayer shows discrete areas of calcium deposition. There are no bone nodules observed in Fig. 1E. However, at this time point there were small nodules present (not shown here). 


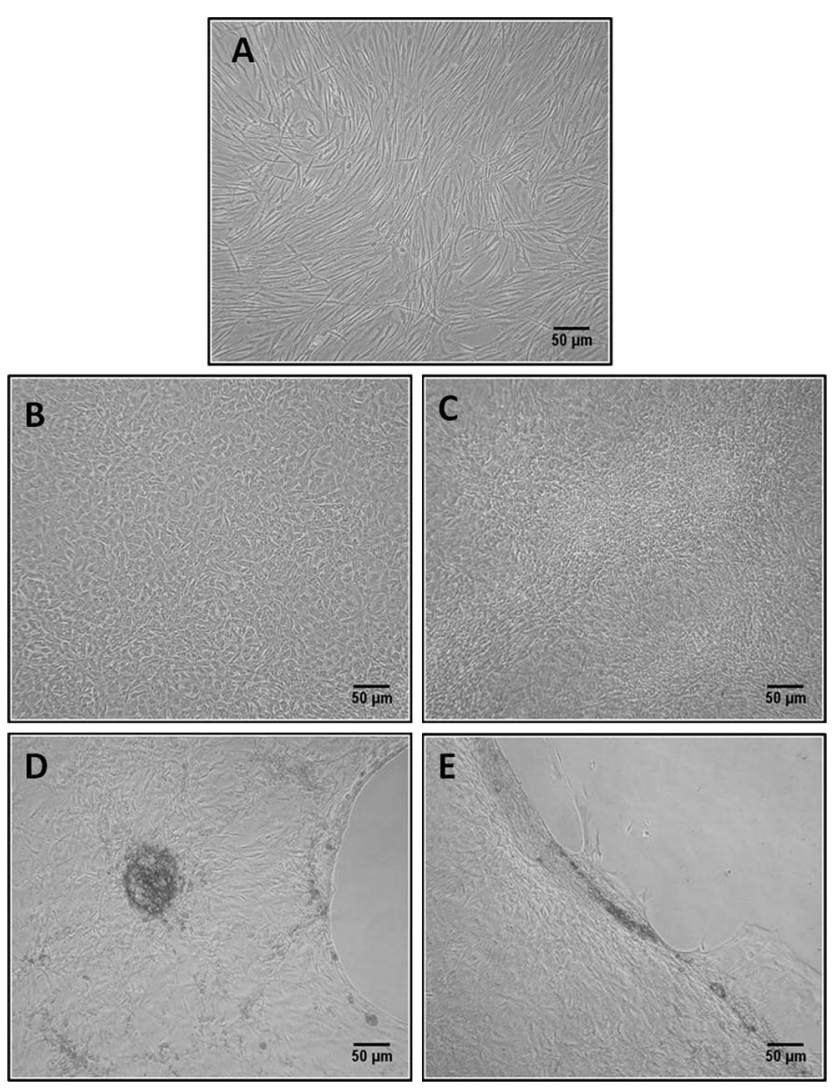

Fig. 1 Phase contrast micrographs of HOBs cultured for (A) 28 days in non-osteogenic media, (B) 7 days, (C), 14 days, (D) 21 days and (E) 28 days in osteogenic media. The scale bar represents $50 \mu \mathrm{m}$.

Significant differences were observed in the growth pattern of U20Ss over the 28 day culture period of non-osteogenic and osteogenic culture. Fig. 2 shows U20Ss cultured for 7 days in (A) non-osteogenic and (B) osteogenic media. At day 7, the
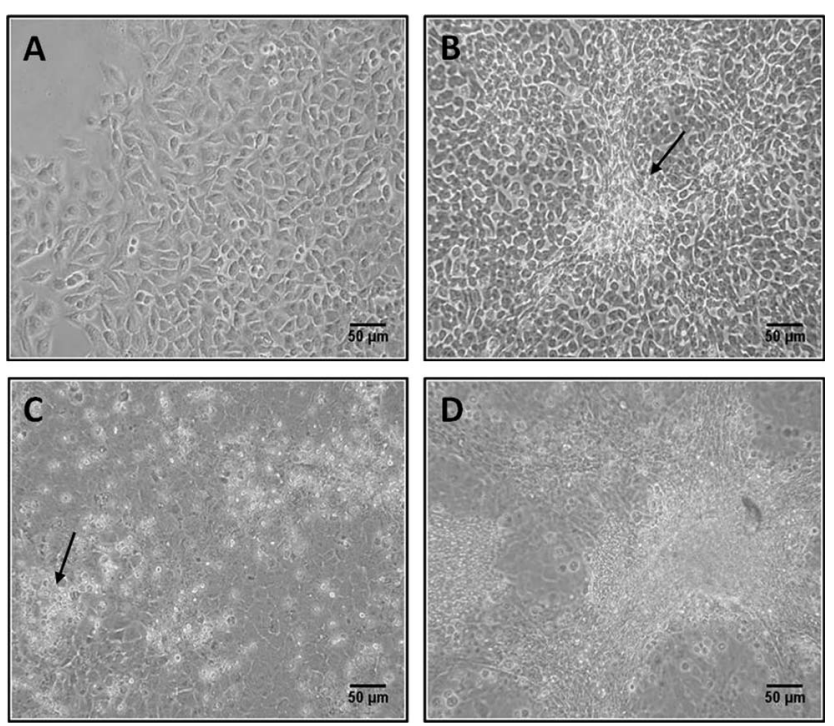

Fig. 2 Phase contrast micrographs of U20Ss cultured in non-osteogenic media for (A) 7 days, (C) 14 days and U20Ss cultured in osteogenic media for (B) 7 days and (D) 14 days. The scale bar represents $50 \mu \mathrm{m}$. osteogenic U20Ss exhibit no apparent changes in cell morphology. However, there are areas of calcium deposition within the matrix, as indicated in Fig. 2B. When Fig. 2A-B is compared with the day 14 samples Fig. 2 C-D, significant changes in the growth patterns of both cultures are apparent. The U20Ss cultured in non-osteogenic media for 14 days appear to exhibit the typical characteristics of a mineralising culture. This is due to there being discrete areas of mineral deposition present in the monolayer, as indicated in Fig. 2C. At day 14 of the U20Ss cultured in osteogenic media, Fig. 2D, there are large areas of mineral deposition in the extracellular matrix and also in the areas surrounding the mineralised regions there is evidence that U20Ss are actively proliferating. This is due to the U20Ss maintaining a cell monolayer that surrounds the large mineral deposits present as shown in Fig. 2D.

Fig. $3 \mathrm{~A}-\mathrm{F}$, shows the phase contrast micrographs of U20Ss in the latter stages of culture in both non-osteogenic (Fig. $3 \mathrm{~A}, \mathrm{C}$ and E) and osteogenic media (Fig. 3 B, D and F). In the samples cultured in non-osteogenic media for 21,25 and 28 days, respectively, there is evidence of accelerated mineralisation of the matrix in large areas of the cell monolayer. At the same time points, the cells cultured in osteogenic media aggressively produced bone nodules, which is a trait that is not normally associated with U20Ss. ${ }^{24}$ The areas of the mineralised matrix, observed in Fig. 3 (B, D and F), appear to be substantially more compact than what is commonly observed with other similar cell
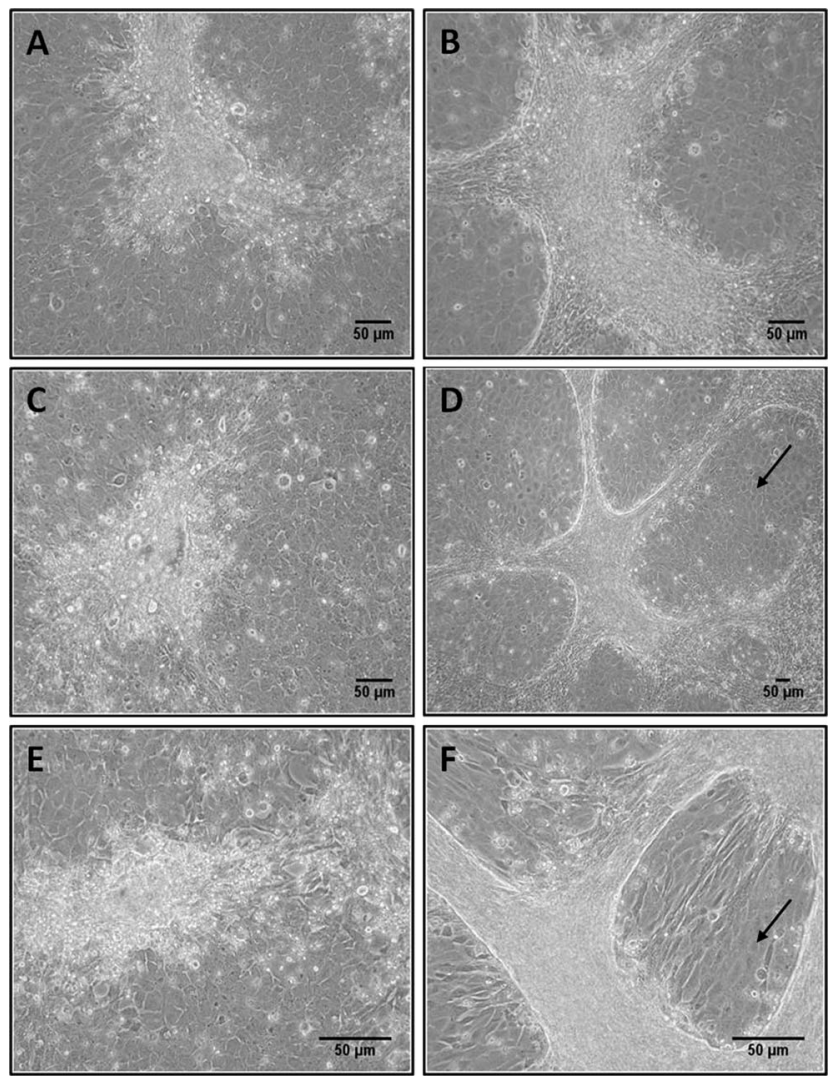

Fig. 3 Phase contrast micrographs of U20Ss cultured in non-osteogenic media for (A) 21 days, (C) 25 days, (E) 28 days and U20Ss cultured in osteogenic media for (B) 21 days, (D) 25 days and (F) 28 days. The scale bar represents (A-F) $50 \mu \mathrm{m}$. 
types, and the areas are interconnected with a dense middle region and dense elongated bridges that are forming the gaps. In comparison with Fig. 2D, there is also evidence of active proliferation of the U20Ss, as indicated by the black arrow.

To quantify the extent of mineralisation of HOBs and U20Ss, alizarin red staining was performed on both cell types cultured in both non-osteogenic and osteogenic conditions for 7, 14, 21 and 28 days using phase contrast microscopy. No appreciable changes were observed in the non-osteogenic cell cultures over the 28 day culture period. However, this was expected due to the lack of change in cell morphology over the same culture period. In work previously published, Gentleman et al. observed nodule formation from primary osteoblasts which occurred in discrete patches throughout culture. ${ }^{26}$ This is reflected in the alizarin red staining of HOBs after 28 days in culture, as shown in Fig. 4 A-D. The osteogenic cell cultures underwent a differing growth pattern over the 28 day culture period, as expected due to the morphological changes observed. HOBs cultured for up to 28 days in osteogenic media revealed positive staining for calcium deposition, which increased steadily through each 7 day interval and at day 28 , discrete patches of mineral deposits are evident (Fig. 4 A-D).

Conversely, throughout the 28 day culture period, the nonosteogenic U20S samples exhibited a growth pattern that is typical of that observed during mineralisation (Supplementary Figure $2 \mathrm{~A}-\mathrm{D} \dagger$ ). Generally, there are small areas present in the monolayer that show signs of mineralisation of the matrix, which are indicated by the positive alizarin red staining. The osteogenic cell culture underwent a similar growth pattern. U20Ss cultured for up to 28 days in osteogenic media revealed significant positive alizarin red staining for calcium deposition, which increases rapidly through each 7 day interval up to day 28 (Fig. 5 A-D). At this time point, large dense areas of mineral deposits are evident, as shown in Fig. 5D.

Quantification of alizarin red staining was performed by an alizarin red colorimetric assay using the cetylpyridinium chloride
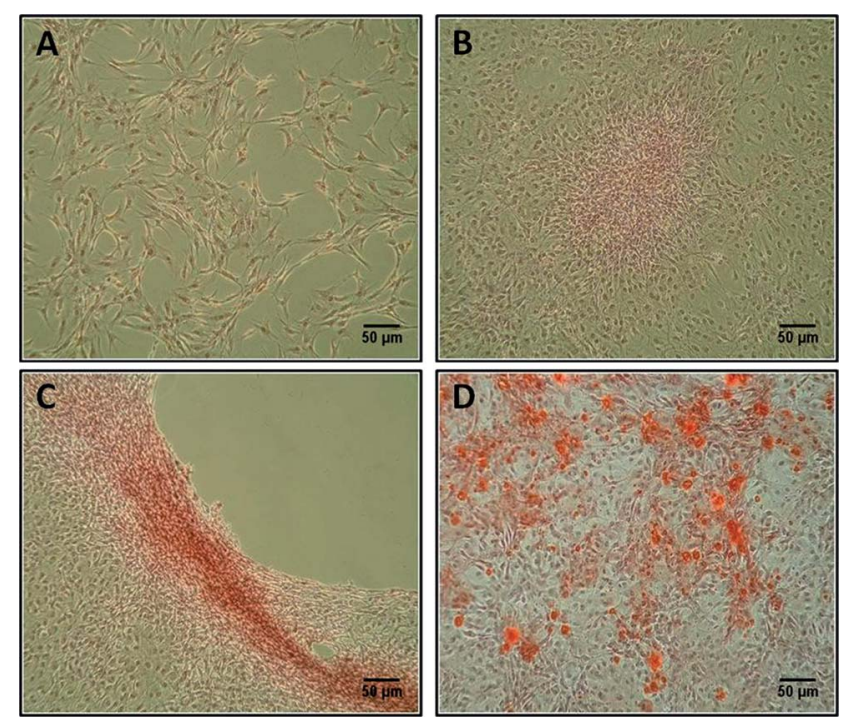

Fig. 4 Alizarin red staining of HOBs cultured in osteogenic media for (A) 7 days, (B) 14 days, (C) 21 days and (D) 28 days. The scale bar represents (A-D) $50 \mu \mathrm{m}$.
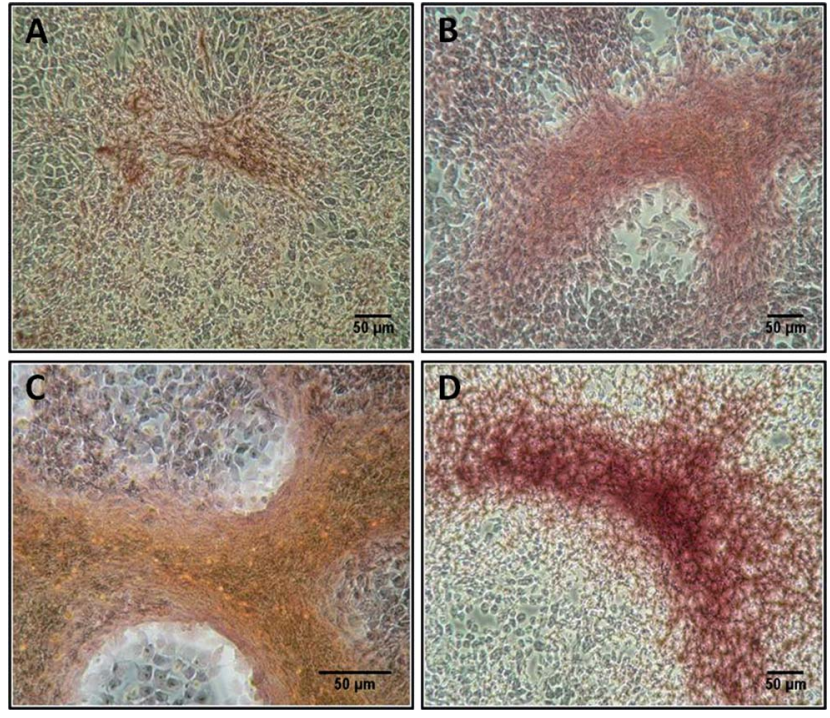

Fig. 5 Alizarin red staining of U20Ss cultured in osteogenic media for (A) 7 days, (B) 14 days, (C) 21 days and (D) 28 days. The scale bar represents (A-D) $50 \mu \mathrm{m}$.

extraction method. Cells were cultured for the full 28 day culture period, with samples taken at 7 day intervals. Supplementary Figure 3 shows the quantification of both non-osteogenic and osteogenic HOBs and U20Ss cultured for up to 28 days. $\dagger$ An increase in alizarin red staining of HOBs cultured in non-osteogenic media is observed between days $7-14$, and in the osteogenic samples as cell proliferation decreases and differentiation increases during days $21-28$, the alizarin red staining increased substantially. Supplementary Figure 3 also shows that alizarin red staining of U20Ss cultured in non-osteogenic media increases significantly throughout the 28 culture period. $\uparrow$ The non-osteogenic U20Ss produced a maximum alizarin red staining at day 28 , represented by a value of 0.56 , and the minimum was at day 7 with a value of 0.15 . In comparison to the results obtained for the non-osteogenic U20Ss, the alizarin red staining of the osteogenic samples increased as the time in culture increased. The lowest amount of alizarin red staining was measured at day 7 with a value of 0.33 . A statistical increase was observed at day 28 of culture with a value of 0.93 . Based on the changes observed in the growth pattern during the 28 day period, it was expected that alizarin red staining would be significantly higher in the osteogenic cultures. However, the non-osteogenic U20Ss had positive alizarin red staining at higher levels at day 21 that plateaued between days 21-28. Despite having lower levels of alizarin red staining at day 28 in non-osteogenic culture, due to the observations made in cell morphology, these results follow the expected pattern and highlight that there is extensive mineralisation in the U20S cultures.

\section{Single cell characterisation of human primary osteoblasts and U20S osteoblast-like cells}

The averaged pre-processed Raman spectra of a single HOB cell and U20S cell are shown in Fig. 6 (A-B) and are composed of peaks that are representative of all biomolecules present in cells. The Raman spectra of both cell types are represented as protein/ 

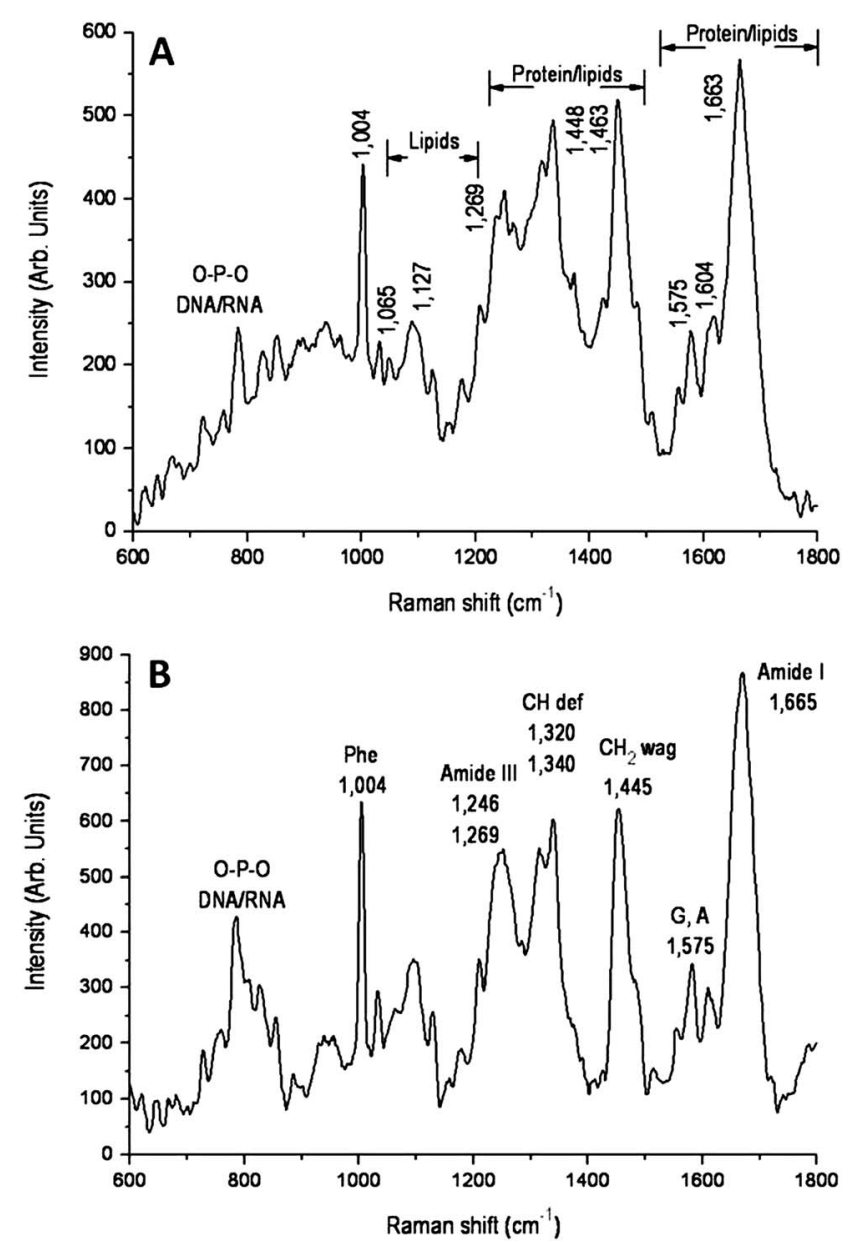

Fig. 6 Raman spectra of (A) HOBs cultured for $48 \mathrm{~h}$ in non-osteogenic media and (B) U20Ss cultured for $48 \mathrm{~h}$ in non-osteogenic media.

lipid dominated signatures with prominent protein bands at $1665 \mathrm{~cm}^{-1}$ (amide I) and $1248 \mathrm{~cm}^{-1}$ (amide III) and strong peaks that are indicative of lipids at Raman frequencies of $720 \mathrm{~cm}^{-1}$ (CN stretching), $985 \mathrm{~cm}^{-1}$ and $1319 \mathrm{~cm}^{-1}$ (CH bending) and $1448 \mathrm{~cm}^{-1}$ ( $\mathrm{CH}$ deformation). There are also well pronounced bands that correspond to the O-P-O backbone stretch $\left(788 \mathrm{~cm}^{-1}\right)$ and, specifically in the U20Ss, the peaks at $826 \mathrm{~cm}^{-1}$ and $857 \mathrm{~cm}^{-1}$, that represent tyrosine ring breathing and O-P-O asymmetric stretching, dominate the $800-900 \mathrm{~cm}^{-1}$ spectral region. The peaks in the region of $750-900 \mathrm{~cm}^{-1}$ are all indicative of the nucleic acid component of a cell and Fig. $6 \mathrm{~B}$ shows that, in U20Ss, the bands in this region are of a higher intensity than those of HOBs, shown in Fig. 6A.

\section{Raman assessment of the osteoinductive potential of human primary osteoblasts and U20S osteoblast-like cells}

After 28 days in culture, dramatic morphological differences were observed in both cell types. In HOBs there were very discrete areas of mineralisation and bone nodule formation. Conversely, in the U20Ss there were large areas of dense mineralised structures. These differences are not reflected in the Raman signal obtained from both cell types, as shown in Fig. 7 (A-B). The time in culture has had little to no effect on the
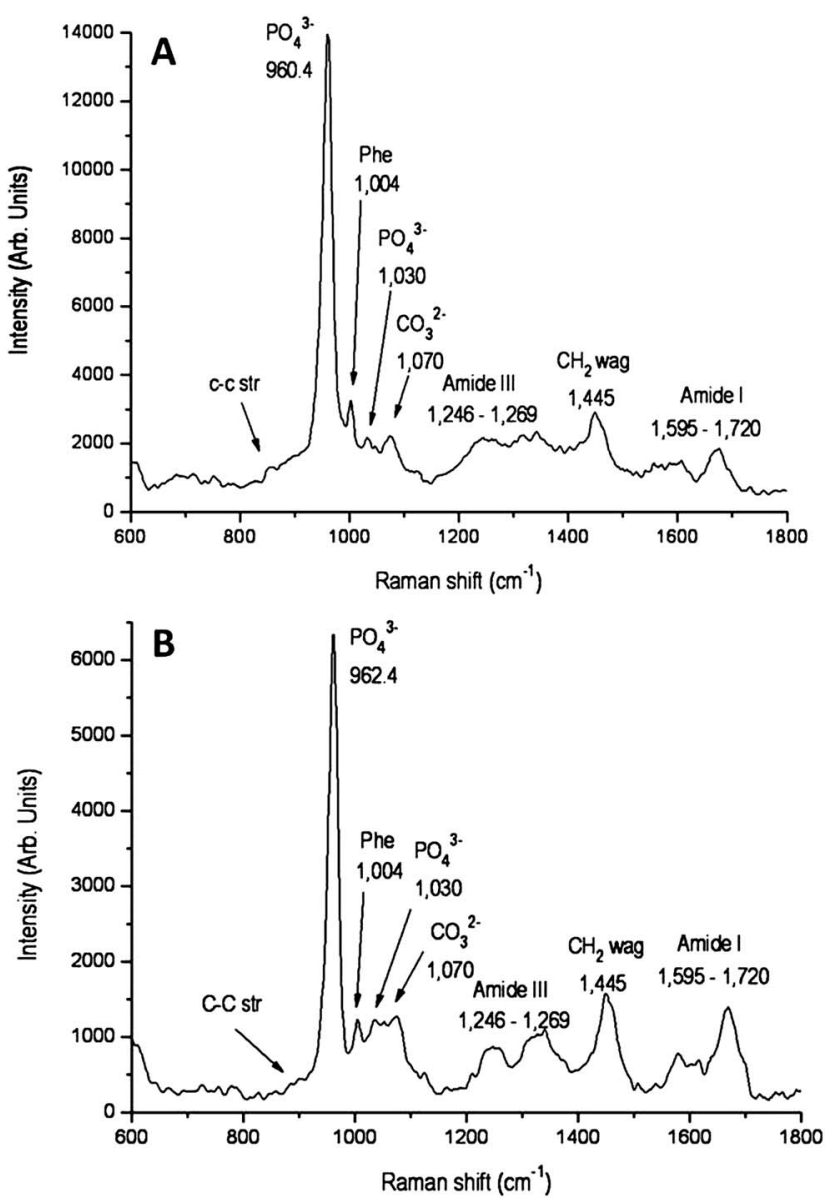

Fig. 7 Raman spectra of (A) HOBs and (B) U20Ss cultured in osteogenic media for 28 days.

Raman spectra obtained at this time point. Both cell types exhibit a Raman signal dominated by peaks that are characteristic of bone material, namely those found at $\sim 960 \mathrm{~cm}^{-1}$, $1030 \mathrm{~cm}^{-1}$ and $1070 \mathrm{~cm}^{-1}$. The HOBs and U20Ss follow the same trend, showing little fluctuation in the results from 28 days, except for a slight shift in the phosphate peak, where in the case of HOBs this peak is at $960.4 \mathrm{~cm}^{-1}$ and in U20Ss it is recorded at $962.4 \mathrm{~cm}^{-1}$. This indicates a small change in mineral species of the mineral produced by the HOBs between days 21 and 28 . However, for HOB samples it is evident that there is a decrease in the $\mathrm{CH}_{2}$ Wag $\left(1445 \mathrm{~cm}^{-1}\right)$ and amide I band $\left(1595-1720 \mathrm{~cm}^{-1}\right)$, which both relate to lipids and proteins respectively.

To confirm the presence of phenotypic markers of osteoblast cells, immunocytochemistry was performed on both cell types after 28 days in culture with non-osteogenic and osteogenic media. Samples were labelled with antibodies to osteopontin $(\mathrm{OPN})$, osteocalcin (OCN) and alkaline phosphatase (ALP). Supplementary Figure 4 (A-F) shows localisation of OPN, OCN and ALP in HOBs cultured for 28 days in (A-C) non-osteogenic media and (D-F) osteogenic media. $\dagger$ Supplementary Figure $5(\mathrm{~A}-\mathrm{F})$ shows localisation of OPN, OCN and ALP in U20Ss cultured in $(\mathrm{A}-\mathrm{C})$ non-osteogenic media and (D-F) osteogenic media for 28 days. $\uparrow$ The characterisation of osteogenic cells is often achieved by examining the expression of OPN, OCN and ALP, which can differ with the stage of differentiation. 
ALP is usually expressed at higher levels at the earlier stages of culture; OPN and OCN are both mid to end stage markers of mineralisation. When comparing Supplementary Figure 4 (A-C) with (D-F) and Supplementary Figure $5(\mathrm{~A}-\mathrm{C})$ with (D-F), it is evident that there is a higher degree of positive staining in the osteogenic samples when compared with the non-osteogenic samples. $\dagger$ These proteins are found only in bone tissue and, as such, this indicates that an extracellular matrix has been produced by the HOBs and U20Ss and over time mineralisation occurred. In the U20S samples cultured in non-osteogenic media (Supplementary Figure $5(\mathrm{~A}-\mathrm{C}) \dagger$ ), there is some evidence of staining of the bone specific proteins, and this can be correlated with the cell morphology findings and the alizarin red results.

The osteoinductive ability of the U20S osteoblast-like cell line described could be a consequence of the addition of osteogenic supplements in the culture medium. As a result of these conditions, the osteoinductive capability of U20Ss was assessed when cultured in normal basal culture medium for 28 days. With regards to U20S osteoblast-like cells, the growth pattern changed over the 28 day period when cultured in non-osteogenic media. The cells maintained their cuboidal osteoblast-like morphology, and there was evidence of calcium deposition in the extracellular matrix. Therefore Raman measurements were acquired at this time point, as shown in Fig. 8. At 7, 14, and 21 days of nonosteogenic culture, the Raman signal was similar to the control spectra (Fig. 6B). However, at day 28 of non-osteogenic culture, the Raman spectrum resembles that of the early stages of the mineralising culture. The spectrum contains peaks that are characteristic of a proteinaceous extracellular matrix with Raman bands at 1246-1269 $\mathrm{cm}^{-1}$ (amide III) and 1595$1720 \mathrm{~cm}^{-1}$ (amide I). The peaks at $788 \mathrm{~cm}^{-1}, 826 \mathrm{~cm}^{-1}$ and $857 \mathrm{~cm}^{-1}$ that are indicative of nucleic acids are also prominent in the spectra. The main interesting feature observed in Fig. 8, is the addition of the peak $\left(962.9 \mathrm{~cm}^{-1}\right)$ that highlights the presence of a hydroxyapatite bone-like mineral in the sample.

Based on these measurements, it can be argued that the structures formed by U20Ss are representative of a bone-like material and that these mineral deposits were produced early in the culture. This is illustrated by the presence of the PO symmetric stretch found at $960 \mathrm{~cm}^{-1}$, indicative of the inclusion

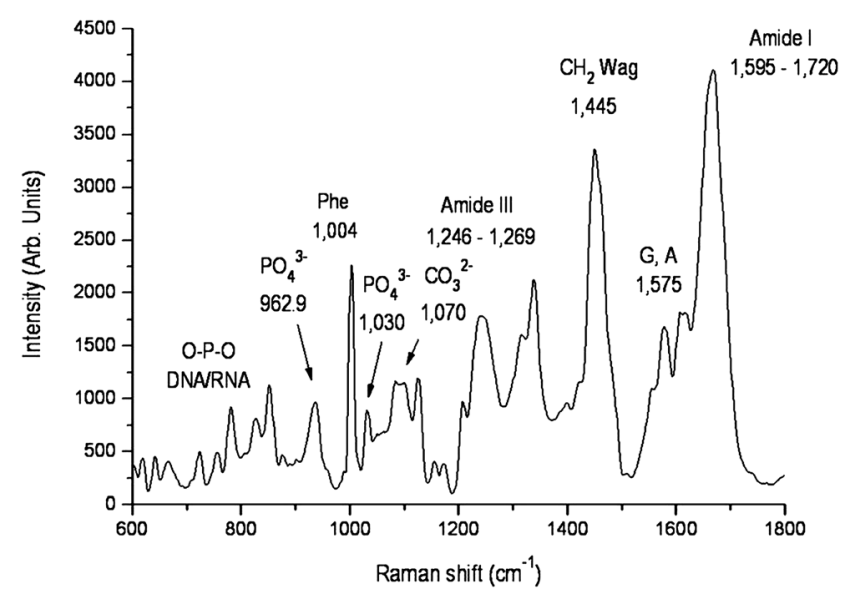

Fig. 8 Raman spectra of U20Ss cultured for 28 days in non-osteogenic media. of hydroxyapatite in the extracellular matrix. It is note-worthy that, to the authors' knowledge, there is little published literature to suggest that U20S osteoblast-like cells produce mineralised nodules with such aggressiveness and density. The results obtained for U20Ss can be compared to another osteosarcoma derived cell lines, namely SaOS-2. Therefore the production of mineralised tissue could simply be due to the high proliferation rate of the U20S cell type. ${ }^{24}$

\section{Univariate peak analysis}

There are many similarities in the Raman spectra obtained from HOBs and U20Ss at day 28. However small changes in the frequency and peak position of the peaks that are characteristic of bone were observed, these were examined using univariate peak analysis. This was performed at 7, 14, 21 and 28 days, in an attempt to determine the mineral-to-matrix ratio and carbonateto-phosphate ratio of the bone-like material produced by both cell types and to provide a comparative analysis of these properties, as shown in Table $1 .{ }^{26-28}$

In order to calculate the mineral-to-matrix ratio, the intensity of the $\mathrm{PO}_{4}{ }^{3-}$ phosphate symmetric stretch was used as the metric of mineral content and the hydroxyproline peak at $\sim 850 \mathrm{~cm}^{-1}$ was used as a measure of the matrix content. ${ }^{26-28}$ Carbonate-tophosphate ratios were calculated according to the methods employed by Gentleman et al. ${ }^{26}$ and Morris et al. ${ }^{29}$ based on the band intensity of the $\mathrm{CO}_{3}{ }^{2-}$ in plane vibrations at $1070 \mathrm{~cm}^{-1}$, and the intensity of the baseline corrected $\mathrm{PO}_{4}{ }^{3-}$ phosphate symmetric stretch at $960 \mathrm{~cm}^{-1}$. Although it has been demonstrated that the overlap between carbonate $\nu_{1}$ and phosphate $\nu_{3}$ bands at $\sim 1071 \mathrm{~cm}^{-1}$ makes it difficult to distinguish between these bands at low carbonate levels $(\sim 0.3 \%)$, at higher carbonate levels $(1-10 \%)$, the ratio of the $1071 \mathrm{~cm}^{-1}$ to $960 \mathrm{~cm}^{-1}$ peak areas were seen to be well correlated with the carbonate content. ${ }^{30}$ Both ratios were calculated from an average of three band intensities and a global average was obtained..$^{26,31-33}$

The mineral-to-matrix ratio measures the amount of mineral in the protein matrix and the carbonate-to-phosphate ratio measures the substitution of carbonate into the apatite lattice. The calculated mineral-to-matrix and carbonate-to-phosphate ratios for primary osteoblasts are shown in Table 1. A linear trend is observed throughout the full 28 day culture period for both the mineral-to-matrix and carbonate-to-phosphate ratios. Hence, as the culture time is increased, the mineral-to-matrix ratio and carbonate-to-phosphate ratio also increased. From the univariate analysis, it is demonstrated that the mineral-to-matrix

Table 1 Carbonate-to-phosphate ratios and mineral-to-matrix ratios as determined by univariate analysis carried out by curve fitting Raman spectra of HOBs and U20Ss cultured for 7-28 days. For each group spectra were recorded from cells in triplicate. Statistically significant difference between Sample $X$ and $\uparrow$ day $7, \ddagger$ day 14, Fr day 21 and $\S$ day 28

\begin{tabular}{|c|c|c|c|}
\hline \multicolumn{2}{|c|}{ Mineral-to-matrix ratio } & \multicolumn{2}{|c|}{ Carbonate-to-phosphate ratio } \\
\hline HOBs & U20Ss & HOBs & U20Ss \\
\hline $1.01 \pm 0.21^{\mathrm{r} \$ \ddagger}$ & $1.09 \pm$ & - & $1.16 \pm 1.02^{\S}$ \\
\hline $7.56 \pm 4.79^{\mathrm{F} \xi \dagger}$ & $2.55 \pm 0.05^{\mathrm{r}}$ & $0.21 \pm 0.06^{\mathrm{F} \S}$ & $1.81 \pm 1.01^{\mathrm{F} \S}$ \\
\hline $14.52 \pm 6.24^{\dagger \dagger}$ & $3.40 \pm 0.09^{\S \dagger}$ & $0.51 \pm 0.34^{8}$ & $2.47 \pm 1.91^{\ddagger}$ \\
\hline $32.39 \pm 8.41^{\text {t十 }}$ & $4.51 \pm 0.18^{\mathrm{rl}+\dagger}$ & $0.67 \pm 0.03^{\ddagger}$ & $3.36 \pm 1.80^{\mathrm{rt}+}$ \\
\hline
\end{tabular}


and carbonate-to-phosphate ratios for both cell types show significant differences. In the case of the primary osteoblasts, the mineral-to-matrix ratio increased with culture time, a trait that has been associated with the differentiation of stem cells into osteoblasts $^{27,34}$ and mineralising calvaria osteoblasts. ${ }^{28,31}$ The carbonate-to-phosphate ratios show a ratio at day 7 that increases significantly through culture time. This highlights that carbonate substitution begins to occur at the earliest stages of culture. Further to this, the values reported here are similar to those calculated for $\mathrm{hMSCs}^{34}$ and they further affirm that carbonate substitution occurs most rapidly between day 14 and 21. Comparatively, the mineral-to-matrix ratio increased 2 fold with culture time, and also reached values that are similar to those reported in previous work. ${ }^{34}$ This confirms that there is mineral present in the protein matrix as expected. Based on this, it can be said that the mineral content of the bone tissue at the later culture time is higher and that the deposition of mineral into the apatite lattice occurs most rapidly during days 21 and 28 .

The calculated mineral-to-matrix and carbonate-to-phosphate ratios for U20S osteoblast-like cells are also shown in Table 1. Notably, while U20Ss follow the same linear pattern of the carbonate-to-phosphate and mineral-to-matrix ratios observed for the primary cell line, substantial differences in the calculated values at the same time points are demonstrated. The carbonateto-phosphate ratios increase with culture time and they reach values that are significantly higher than the primary osteoblasts, indicating that more carbonate has been substituted into the apatite lattice. These results are interesting, as the values are significantly higher than any values reported on the carbonateto-phosphate ratios of mineralising cultures. The carbonate-tophosphate ratios may not be in accordance with findings reported by various research groups. ${ }^{26,28,31,34}$ However, the ratio is similar to the carbonate-to-phosphate ratio of native bone that was found to have a value of $3.84 \pm 0.03$. The mineral-to-matrix ratios calculated for U20Ss were also significantly different when compared with the primary osteoblasts. The maximum ratio after 28 days in culture is $4.51 \pm 0.18$, whereas the ratio of the primary osteoblasts at the same time point is $32.4 \pm 8.4$. This was not expected after the positive similarity of the carbonate-tophosphate ratios to native bone and thus it illustrates that a low amount of mineral has been deposited in the protein matrix in the case of the osteoblast-like U20S cells.

Bone quality can be assessed using univariate peak analysis and in particular, the $\mathrm{PO}_{4}{ }^{3-}$ symmetric band, which is the most intense Raman peak present in the signal from bone tissue, can be used to investigate bone crystallinity. A major aspect when characterising bone material is resolving changes in the phosphate band in terms of both frequency and peak position. Bone mineral crystallinity can be calculated from the full width half maximum (FWHM) of the $\mathrm{PO}_{4}{ }^{3-}$ symmetric band, whereby the lower the FWHM the higher the crystallinity of the material. ${ }^{34}$ The results reported on the primary osteoblast cells used in this study are consistent with those reported in the literature from various research groups. ${ }^{26,35,36}$ This is specifically regarding the ability of the primary osteoblasts to produce bone nodules in small mineralised areas in the culture. However, the results obtained from U20S osteoblast-like cells are interesting to say the least. This is due to the contradictory findings detailed by Anderson et al., Raval et al. and Dass et al. who reported that osteoblast-like U20Ss have no osteoinductive ability as they consistently failed to support the formation of mineralised tissue. $^{22-24}$ On the contrary, in this study it is demonstrated that U20Ss do in fact produce mineralised bone tissue, and they do so very aggressively and in dense multilayered regions of the cell culture. This is in accordance with studies performed by Hunt et al. ${ }^{37}$ who reported that U20Ss expressed higher levels of bone growth and morphogenic factors than other osteosarcoma cell lines that are well characterised as being osteoinductive. ${ }^{37}$

Based on the findings obtained from the univariate peak analysis, it is suggested that the primary osteoblasts and osteoblast-like cells did successfully support the formation of mineralised nodules that are significantly different in composition. As such, the crystallinity of the mineralised nodules is determined using the FWHM of the PO symmetric stretch at $\sim 960 \mathrm{~cm}^{-1}$ as shown in Table 2. Based on this, the primary osteoblasts formed a highly crystalline bone material with the highest degree of crystallinity observed at day 14 with a value of $27.11 \pm 5.93$. An interesting point is that the crystallinity of the older more mature bone (day 28) is higher than the newly formed bone (day 21). This finding could also be due to poorly organised apatite mineral that occurs during active bone remodelling. The findings reported here are consistent with those from a study by Farlay et al. who also found that older bone had a higher crystallinity that new bone tissue. ${ }^{38}$ The crystallinity of the bone material reported here is significantly higher when compared to hMSCs which are from the same origin. ${ }^{34}$ This suggests that the primary osteoblasts produce an apatite mineral that is highly organised, although less so relative to the crystallinity found in the bone material produced by U20Ss. The mineral produced by U20Ss is significantly more crystalline when compared to the primary osteoblasts. The highest degree of crystallinity of U20Ss is observed at day 7 with a value of $14.74 \pm 1.64$. This was not maintained over longer periods and the crystallinity reached a plateau between days 14 and 28. Nevertheless, it is suggested that U20Ss still produced a bone material that was significantly more crystalline than the bone material produced by primary osteoblasts. Another key finding, is that the crystallinity of the U20S bone material can be compared with that of native bone which was found to have a FWHM of $19.17 \pm 5.13 .{ }^{34}$ The results may indicate that the U20S osteosarcoma cell line is not a good model for human osteoblasts, unless one defines a good model as any cell that makes some apatite-like mineral deposited in/on collagen, with measured bone quality markers that are similar to native bone. Nevertheless, U20Ss may have potential to serve as a model for evaluating and understanding composition differences between normal bone and osteoblastic lesions sometimes found when prostate cancer metastasizes to bone. ${ }^{30}$

\section{Principal component analysis}

The results obtained from univariate peak analysis highlighted differences in the composition of the bone material produced over a 28 day period. Principal component analysis (PCA) was performed on the Raman spectra obtained from HOBs and U20Ss cultured in osteogenic media in order to highlight the similarities and/or differences in the data set.

PCA shows the maximum variance within a data set which is then represented by a scatter plot. The $3 \mathrm{D}$ plot comparing the 
Table 2 Phosphate peak analysis and mineral crystallinity as determined by univariate analysis carried out by curve fitting Raman spectra of HOBs and U20Ss cultured for 7-28 days in osteogenic media. For each group spectra were recorded from cells in triplicate and $n$ represents the number of spectra recorded

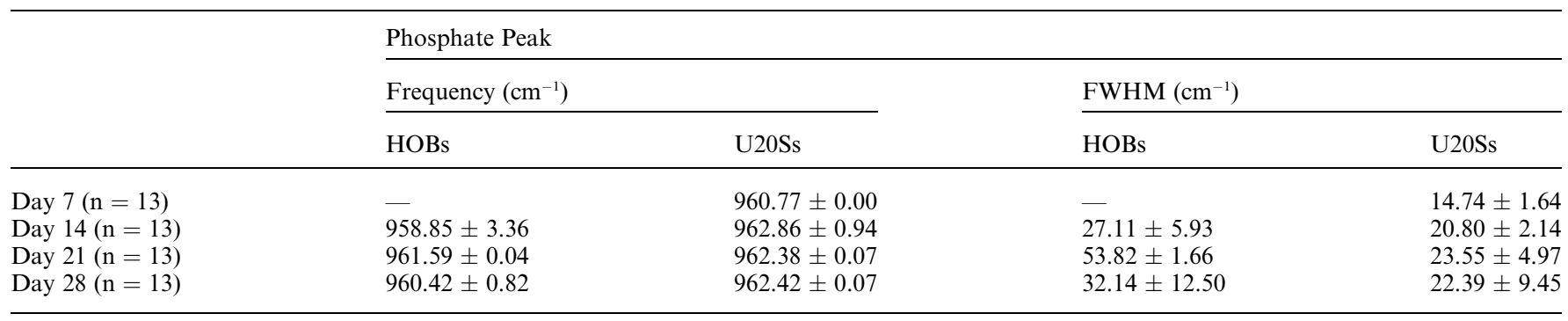

different culture time points for the osteogenic HOBs is displayed in Fig. 9A. The 3D scores plot shows evidence of significant separation between the 7 day intervals and subsequently the scores for these cultures times are compactly clustered. PC1, PC2 and PC3 represent $86 \%, 8 \%$ and $2 \%$ of the variance of the data set, respectively, although group separation appears to be primarily due to PC2. The loading of PC1 demonstrates the separation between day 14 and day 28 . This loading is predominantly composed of a sharp peak at $\sim 960 \mathrm{~cm}^{-1}$. Major
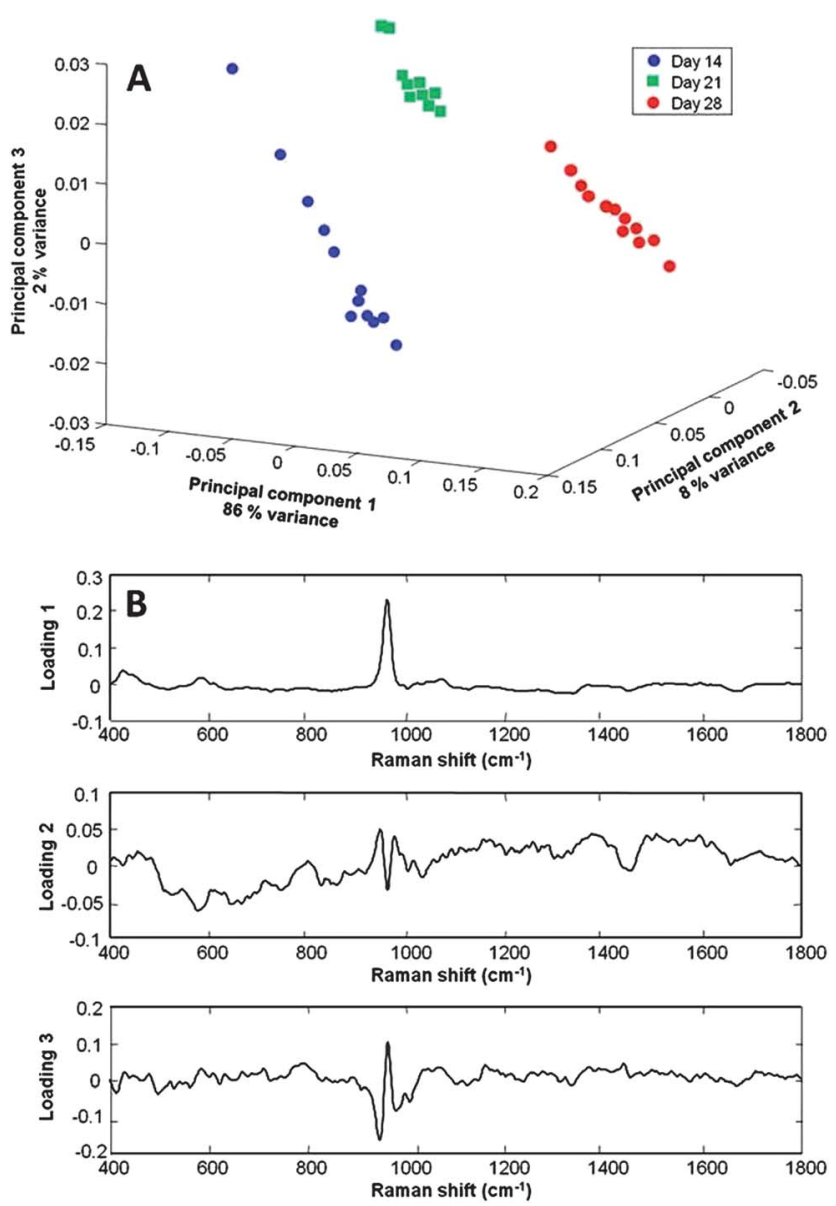

Fig. 9 (A) Scatter plot of the 3 first principal components after PCA was carried out on the Raman spectra recorded from HOBs grown in osteogenic media and (B) shows a plot of the loadings that correspond to the 3 first principal components used for Fig. 9 (A). similarities are observed between the loading of PC1 and the spectra of bone tissue, specifically in the $900-1000 \mathrm{~cm}^{-1}$ spectral range, highlighting the contribution of the mineral species present during the recording of the Raman measurements. The loading of PC2 is a combination of various peaks with different intensities and it is responsible for discriminating between the culture period of the day 14 and days $21-28$. It accounts for $8 \%$ of the variance of the total data set and the loading plot is dominated by spectral features that correspond to the lipid and protein signatures at $1450 \mathrm{~cm}^{-1}$ and $1665 \mathrm{~cm}^{-1}$, respectively. From PC2, it can be seen that there is a negative peak at $960 \mathrm{~cm}^{-1}$ that represents the phosphate mode and a positive band at $1450 \mathrm{~cm}^{-1}$ that relates to the $\mathrm{CH}_{2}$ Wag. PC3 represents only $2 \%$ of the variance, and as such it provides evidence of slight intragroup variability in day 14 samples, due to the variation in the mineral species present at that time point.

As highlighted previously, the use of an osteoblast-like cell line as a model for primary osteoblasts specifically with regards to the osteoinductive ability is not fully understood. U20Ss were cultured for a 28 day period in osteogenic media and data preprocessing was performed, as was the case for the HOBs. The $3 \mathrm{D}$ plot acquired from the U20Ss after PCA and the corresponding loading plots are displayed in Fig. 10A-B, respectively.

U20Ss demonstrated a different pattern in the 3D scores plot. The 3D plot shows that the results obtained from the PCA of the Raman spectra at day 7-14 of culture exhibit few distinguishing differences between the spectra from the two time points as the two groups are overlapping. Once again, the measurements taken at day 21 and day 28 are consistent with those obtained from the HOBs in that a clear separation is observed at days 21 and 28 . PC1 represents $64 \%$ of the total variance and this is due to positive peaks in the areas that represent both proteins and lipids, including in the 1246-1269 $\mathrm{cm}^{-1}, 1319-1340 \mathrm{~cm}^{-1}$, $1445 \mathrm{~cm}^{-1}, 1520-1720 \mathrm{~cm}^{-1}$ spectral regions and to the positive loading at $\sim 960 \mathrm{~cm}^{-1}$ that corresponds to the phosphate symmetric stretch. PC2 demonstrates the separation between day 21 and day 28 and PC3 represents the separation between day 21 and the combined groups of days 7, 14 and 28. PC2 accounts for $19 \%$ of the explained variance and with regards to the loading plot it is related to the positive peak representing the mineral species and the negative peaks corresponding to proteins/lipids. PC3 represents $6 \%$ of the variance and is again related to the mineral species in the Raman spectra. This indicates that due to the positive loadings in $\mathrm{PC} 1$ that reflect proteins/lipids, PC1 causes separation between days 7-14 from 21-28 and PC2 causes 


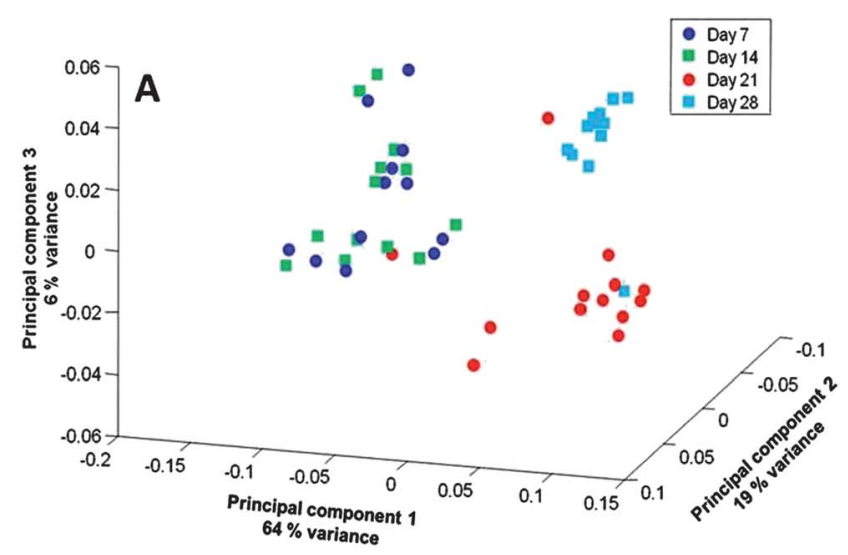

found here are in good agreement with those observed by Stewart et al., and while all of them are markers and precursors of hydroxyapatite, the fact that there are different mineral species indicates the variability of the bone material produced by these cell types when cultured under the same conditions for the same period of time. ${ }^{32}$

\section{Conclusion}

When considering the use of cell lines and in particular osteosarcoma derived cell lines as an alternative to primary cells, there are many issues that need to be addressed. On one hand, osteosarcoma derived cells rapidly proliferate and, because they are immortalized, they maintain their phenotype. On the other hand, primary osteoblast cells are challenging to culture and with longer culture times they may become phenotypically unstable. ${ }^{10}$ However, the major shortcomings of the osteosarcoma cell line are 1) that it is derived from a bone tumor that is consistently known to have a very aggressive growth pattern and 2) it may not be a true representation of human primary cells of the same origin. In this regard, both cell types produced similar patterns in their Raman signal, although one significant difference was observed. Spectral analysis of the osteosarcoma cells showed an increased concentration of nucleic acids and lower concentration of proteins in contrast to the human primary osteoblasts which showed the opposite.

The non-osteoinductive ability of osteosarcoma derived U20Ss is controversial to say the least and as a consequence it has been examined and compared with human primary osteoblasts in this study. In order to do this, various biological, univariate, multivariate techniques were employed in parallel with Raman spectroscopy to provide a comprehensive comparison of the cell types.

Primary osteoblasts produced a bone mineral in discrete areas of the osteogenic culture. This began from day 7 and increased throughout the 28 day culture period, while all other parameters remained the same throughout. This cell type is commonly used to investigate the capability to produce mineralised nodules. However, in terms of culture methods they are not straight forward to expand in vitro. U20S osteoblast-like cells, which are the proposed alternative, also produced a bone material in the osteogenic culture environment over the 28 day period. U20Ss also produced mineralised nodules when in a non-osteogenic environment. The mineralised tissue produced by primary cells is more commonly compared with native bone, but in this case while the material is similar to that formed by differentiated hMSCs, ${ }^{34}$ based on these findings it can be said that it is less comparable to native bone. Here it has been shown that the mineralised structures formed by the osteoblast-like U20Ss has similar attributes to native bone, i.e. carbonate-to-phosphate ratio, mineral-to-matrix ratio and crystallinity. This observation is quite surprising considering not only the origin of these cells but also the crucial fact that they have been consistently reported to have no osteoinductive ability. To the best of the author's knowledge, this is the first time to date that the bone-like material produced by U20Ss has been closely analysed by Raman spectroscopy and univariate analysis in an attempt to give an overall understanding using the properties that are commonly employed to determine bone quality. Although the results may indicate that 
the U20S osteosarcoma cell is not a good model for human osteoblasts, they may have potential to serve as a model for composition differences between normal bone and osteoblastic lesions.

\section{Acknowledgements}

This work was funded in part by the Cross Border Research and Development Funding Programme - Strengthening the AllIsland Research Base (Grant Number UU005). This research was also supported in part by the National Biophotonics and Imaging Platform (NBIP) Ireland funded under the Higher Education Authority PRTLI (Programme for Research in Third Level Institutions) Cycle 4, co-funded by the Irish Government and the European Union Structural fund.

\section{References}

1 R. Langer and J. P. Vacanti, Tissue engineering, Science, 1993, 260(5110), 920-926.

2 J. E. Aubin, Bone stem cells, Journal of Cellular Biochemistry, 1998, 73-82.

3 P. Ducy and G. Karsenty, The family of bone morphogenetic proteins, Kidney Int, 2000, 57, 2207-2214.

4 C. A. Gregory, et al. An Alizarin red-based assay of mineralization by adherent cells in culture: comparison with cetylpyridinium chloride extraction, Analytical Biochemistry, 2004, 329(1), 77-84.

$5 \mathrm{H}$. Nakamura, Morphology, Function, and Differentiation of Bone Cells, Journal of Hard Tissue Biology, 2007, 16(1), 15-22.

6 P. O'Hare., The Role of Inner Pore Topography on Osteoblast Response in Hydroxyapaite Scaffolds. Ph.D Thesis, 2006.

7 I. Notingher, et al. In situ non-invasive spectral discrimination between bone cell phenotypes used in tissue engineering, Journal of Cellular Biochemistry, 2004, 92(6), 1180-1192.

8 R. J. Swain, et al. Assessment of Cell Line Models of Primary Human Cells by Raman Spectral Phenotyping, Biophysical Journal, 2010, 98(8), 1703-1711.

9 J. W. Chan, et al., Micro-Raman Spectroscopy Detects Individual Neoplastic and Normal Hematopoietic Cells. 2006. 90(2). pp. 648656.

10 D. Rubio, et al. Molecular Characterization of Spontaneous Mesenchymal Stem Cell Transformation, PLoS ONE, 2008, 3(1), e1398.

11 B. D. Boyan, et al. Osteoblast-Mediated Mineral Deposition in Culture is Dependent on Surface Microtopography, Calcified Tissue International, 2002, 71(6), 519-529.

12 B. D. Boyan, et al. Mechanisms Involved in Osteoblast Response to Implant Surface Morphology, Annual Review of Materials Research, 2001, 31(1), 357-371.

13 B. D. Boyan, et al. Surface roughness mediates its effects on osteoblasts via protein kinase A and phospholipase A2, Biomaterials, 1999, 20(23-24), 2305-2310.

$14 \mathrm{~S}$. Schmitt, et al. Comparative in vitro study of the proliferation and growth of ovine osteoblast-like cells on various alloplastic biomaterials manufactured for augmentation and reconstruction of tissue or bone defects, Journal of Materials Science: Materials in Medicine, 2008, 19(3), 1441-1450.

$15 \mathrm{G}$. Bilbe, et al. PCR phenotyping of cytokines, growth factors and their receptors and bone matrix proteins in human osteoblast-like cell lines, Bone, 1996, 19(5), 437-445.

16 J. Clover and M. Gowen, Are MG-63 and HOS TE85 human osteosarcoma cell lines representative models of the osteoblastic phenotype?, Bone, 1994, 15(6), 585-591.

17 G. Jell, et al. Bioactive glass-induced osteoblast differentiation: A noninvasive spectroscopic study, Journal of Biomedical Materials Research Part A, 2008, 86A(1), 31-40.
18 J. E. Gough, I. Notingher and L. L. Hench, Osteoblast attachment and mineralized nodule formation on rough and smooth $45 \mathrm{~S} 5$ bioactive glass monoliths, Journal of Biomedical Materials Research Part A, 2004, 68A(4), 640-650.

19 G. Jell, I. Notingher and O. Tsigkou, Raman spectroscopic determination of foetal osteoblast differentiation following exposure to Bioglass 45S5 dissolution ions, Tissue Eng, 2005.

20 I. Notingher and L. L. Hench, Study of Osteoblasts Mineralisation In vitro by Raman Microspectroscopy, Key Engineering Materials, 2004, 254-256, 769-772.

21 A. Kumarasuriyar, et al. Glycosaminoglycan composition changes with MG-63 osteosarcoma osteogenesis in vitro and induces human mesenchymal stem cell aggregation, Journal of Cellular Physiology, 2009, 218(3), 501-511.

$22 \mathrm{H}$. C. Anderson, et al. The bone inducing agent in SA0S-2 cell extracts and secretions, Cells and Materials, 1998, 8, 89-98.

23 P. Raval, et al. Expression of bone morphogenetic proteins by osteoinductive and non-osteoinductive human osteosarcoma cells, Journal of dental research, 1996, 75(7), 1518.

24 C. Dass, et al. A novel orthotopic murine model provides insights into cellular and molecular characteristics contributing to human osteosarcoma, Clinical and Experimental Metastasis, 2006, 23(7), $367-380$

$25 \mathrm{~F}$. Bonnier, et al. Imaging live cells grown on a three dimensional collagen matrix using Raman microspectroscopy, Analyst, 2010, 135(12), 3169-3177.

26 E. Gentleman, R. J. Swain, N. D. Evans, S. Boonrungsiman, G. Jell, M. D. Ball, T. A. V. Shean, M. L. Oyen, A. Porter and M. M. Stevens, Comparative materials differences revealed in engineered bone as a function of cell-specific differentiation, Nat Mater, 2009, 8(9), 763-770.

27 N. J. Crane, A Nondestructive method for monitoring in vitro stem cell osteogenic differentiation with Raman spectroscopic monitoring, Raman Spectroscopy application note (PerkinElmer), 2009.

28 C. P. Tarnowski, M. A. Ignelzi and M. D. Morris, Mineralization of Developing Mouse Calvaria as Revealed by Raman Microspectroscopy, Journal of Bone and Mineral Research, 2002, 17(6), 1118-1126.

29 M. Morris and G. Mandair, Raman Assessment of Bone Quality, Clinical Orthopaedics and Related Research $\AA$, 2011, 469(8), 21602169.

30 A. Awonusi, M. Morris and M. Tecklenburg, Carbonate Assignment and Calibration in the Raman Spectrum of Apatite, Calcified Tissue International, 2007, 81(1), 46-52.

31 M. D. Morris, et al. Early mineralization of normal and pathologic calvaria as revealed by Raman spectroscopy. 2002: SPIE.

$32 \mathrm{~S}$. Stewart, et al. Trends in early mineralization of murine calvarial osteoblastic cultures: a Raman microscopic study, Journal of Raman Spectroscopy, 2002, 33(7), 536-543.

$33 \mathrm{H}$. K. Chiang, et al. In situ Raman spectroscopic monitoring of hydroxyapatite as human mesenchymal stem cells differentiate into osteoblasts, Journal of Raman Spectroscopy, 2009, 40(5), 546549.

34 L. L. McManus, et al. Raman spectroscopic monitoring of the osteogenic differentiation of human mesenchymal stem cells, Analyst, 2011, 136(12), 2471-2481.

$35 \mathrm{H}$. Ohgushi, et al. In vitro bone formation by rat marrow cell culture, J. Biomed. Mater. Res., 1996, 32(3), 333-340.

36 H. Collignon, M. J. Davicco and J. P. Barlet, Isolation of Cells from Ovine Fetal Long Bone and Characterization of their Osteoblastic Activities during in vitro Mineralization, Arch. Physiol. Biochem., 1997, 105(2), 158-166.

37 T. R. Hunt, J. R. Schwappach and H. C. Anderson, Healing of a Segmental Defect in the Rat Femur with Use of an Extract from a Cultured Human Osteosarcoma Cell-Line (Saos-2). A Preliminary Report, J Bone Joint Surg Am, 1996, 78(1), 41-8.

38 D. Farlay, et al. Mineral maturity and crystallinity index are distinct characteristics of bone mineral, J. Bone Miner. Metab., 2010, 28(4), 433-445. 